\title{
Magnetic Force Microscopic Images of Nanometer-Sized Polyradical Particles
}

\author{
Tsuyoshi MichinObU, Jun InUi, and Hiroyuki NiSHIDE ${ }^{\dagger}$ \\ Department of Applied Chemistry, Waseda University, Shinjuku, Tokyo 169-8555, Japan
}

(Received September 17, 2002; Accepted November 6, 2002)

\begin{abstract}
Nanometer-sized polymer particles bearing 2,2,6,6-tetramethylpiperidine-1-oxyl (TEMPO) radicals were prepared. The emulsifier-free emulsion polymerization of methacrylic acid, methyl methacrylate, and methylenebisacrylamide and the following introduction of the TEMPO moiety produced polyradical particles with different spin concentrations. The TEMPO-combined particle was also prepared in one step by the anionic dispersion polymerization of 4-methacryloyloxy-2,2,6,6-tetramethylpiperidine-1-oxyl. The position and horizontal shape in the atomic and magnetic force microscopic images of the polyradical particle coincided with each other. The vertical scale of the magnetic image responded to the spin concentration of the particle. The particle bearing the radicals on its surface gave a hollow dot image.

KEY WORDS Polyradical / Microparticle / Magnetic Force Microscopy (MFM) / Emulsifier-Free Emulsion Polymerization / Anionic Dispersion Polymerization /
\end{abstract}

In recent years, magnetic dots consisting of metals or metal oxides of $10-100 \mathrm{~nm}$ sizes have been significantly investigated because of their potential use as future high-density magnetic recording media. ${ }^{1}$ However, there has been no report, to our knowledge, on a magnetically responsible "dot" composed of purelyorganic derived materials. Among the nm-sized materials, organic polymers that possess a single molecularbased size in the nanometer range have received considerable attention, because they are prepared by conventional polymer chemistry and their molecular structure is designable. ${ }^{2}$ In particular, the preparation of organic polymer-based particles with a size from $10 \mathrm{~nm}$ to $\mu \mathrm{m}$ has been established and their applications have been widely investigated. ${ }^{3}$ The emulsion and dispersion polymerizations are useful for preparing polymer particles in that size range.

One of the ways to make non-diamagnetic and paramagnetic organic macromolecules is the accumulation of stable open shell molecules by preparing the polymer bearing stable radicals pendantly or integrating them into the main chain. ${ }^{4}$ They are often called polyradicals and have been studied as a redox polymer and an antioxidant. Nevertheless, no attempt has been made using a nm-sized polyradical particle composed of persistent organic radical species up to now (We have already reported $\pi$-conjugated but non-Kekulé-type and highspin polyradicals with a nm-size, but they were not persistent under air at room temperature $\left.{ }^{5}\right)$. We selected, in this study, the 2,2,6,6-tetramethylpiperidine-1-oxyl (TEMPO) radical as a persistent radical, and describe the preparation of nm-sized and single molecular-based

\footnotetext{
${ }^{\dagger}$ To whom correspondence should be addressed.
}

particles bearing TEMPO radicals and the detection of a very weak magnetic signal of the polyradical particles by magnetic force microscopy (MFM) as a dot image.

\section{EXPERIMENTAL}

\section{Synthetic Procedures}

An emulsifier-free emulsion polymerization was carried out in a $300 \mathrm{~mL}$ round-bottom flask equipped with a reflex condenser, a nitrogen inlet, and an extendable bladed agitator. The ratio of monomers to water was held constant at $6 / 94(\mathrm{~g} / \mathrm{g})$. The monomer feed composition, methyl methacrylate/methacrylic acid/methylenebisacrylamide, was changed from $94 / 1 / 5$ to $65 / 30 / 5 \mathrm{~mol} \%$. The weighed monomers and water, e.g., $4.0 \mathrm{~g}$ of methyl methacrylate, $1.55 \mathrm{~g}$ of methacrylic acid, and $0.45 \mathrm{~g}$ of methylenebisacrylamide in $89 \mathrm{~mL}$ of water, were put into the flask and nitrogen was bubbled into the solution for $30 \mathrm{~min}$ at $80^{\circ} \mathrm{C}$ with stirring at $300 \mathrm{rpm}$. An aqueous solution of $0.022 \mathrm{~g}$ potassium persulfate $(5 \mathrm{~mL})$ was added to initiate the polymerization. The polymerization was continued for $4 \mathrm{~h}$ and then allowed to cool. The produced polymer particle was purified with water by three centrifugation separations at $12000 \mathrm{rpm}$ for $20 \mathrm{~min}$, and dried in vacuo at $100^{\circ} \mathrm{C}$ for $24 \mathrm{~h}$. A $0.1 \mathrm{~g}$ sample of the particles was then dispersed into $10 \mathrm{~mL}$ of thionyl chloride and heated to $70^{\circ} \mathrm{C}$ for $24 \mathrm{~h}$. After thoroughly removing the thionyl chloride, $0.1 \mathrm{~g}$ of 4-amino-2,2,6,6-tetramethylpiperidine-1-oxyl and $3 \mathrm{~g}$ of triethylamine in $5 \mathrm{~mL}$ of dichloromethane were added and stirred at room temperature for $24 \mathrm{~h}$. The polyradical particle was isolated by repeated decanta- 
tion, washing with dichloromethane, and drying in vacuo.

The anionic dispersion polymerization of 4methacryloyloxy-2,2,6,6-tetramethylpiperidine-1-oxyl (MOTMP) was carried out in a Schlenk tube under an inert atmosphere. A typical procedure is as follows. In the tube were directly placed $5 \mathrm{~mL}$ of anhydrous hexane from the distillation apparatus, $0.2 \mathrm{~g}$ of MOTMP, and $0.02 \mathrm{~g}$ of polystyrene-block-polybutadiene, and the mixture was stirred until dissolved. A $0.2 \mathrm{~mL}$ hexane solution of sec-butyllithium $(1.0 \mathrm{M})$ was added dropwise with a dried syringe at room temperature. After stirring for $30 \mathrm{~min}$, the reaction was terminated with methanol.

Linear polyradicals, poly(4-methacryloylaminoand 4-methacryloyloxy-2,2,6,6-tetramethylpiperidine1-oxyl), were prepared according to the literature. The former was obtained by the chemical oxidation of the precursor polymer with hydrogen peroxide in the presence of ethylenediaminetetraacetic acid and sodium tungstate $\left(0.22\right.$ spin unit ${ }^{-1} ; 0.91 \mathrm{mmol} \mathrm{g}^{-1}$, $[\eta]=1.7$ in acetone at $20^{\circ} \mathrm{C}$. The latter was obtained by the anionic polymerization of MOTMP with secbutyllithium in THF at room temperature $(0.81$ spin unit $\left.^{-1} ; 3.3 \mathrm{mmol} \mathrm{g}^{-1}, M_{\mathrm{n}}=2.3 \times 10^{4}\right)$.

The polystyrene-block-poly(methyl methacrylate) was prepared by the living radical polymerization of methyl methacrylate using the chlorine atom endcapped polystyrene and $\mathrm{CuCl}$ and 2, 2' -bipyridine as an initiator at $130^{\circ} \mathrm{C}\left(25 \mathrm{wt} \%\right.$ polystyrene content, $M_{\mathrm{n}}=$ $\left.9.6 \times 10^{4}\right){ }^{7}$

\section{Materials}

Polystyrene-block-polybutadiene was purchased from Aldrich and was purified by repeated reprecipitation from chloroform into methanol (30 $\mathrm{wt} \%$ polystyrene content, $M_{\mathrm{n}}=1.7 \times 10^{5}$ ). Methacrylic acid and methyl methacrylate were purified by distillation under reduced pressure before use. The potassium persulfate was recrystallized from water. The solvents for the anionic dispersion polymerization were carefully distilled under nitrogen. The solvents and triethylamine were purified in the usual manner. The other reagents were used as received.

\section{Measurements}

Magnetic susceptibility was measured using a Quantum Design MPMS SQUID magnetometer over 2$300 \mathrm{~K}$ in a field of $5000 \mathrm{G}$. The spin concentration of the polyradical particles was estimated from the Curie constant of $\chi_{\mathrm{g}}-T$ plot.

Atomic force microscopy (AFM) and MFM measurements were performed using a Nanoscope IIIa mul- timode AFM/MFM microscope (Digital Instruments Inc.). A drop of dilute dichloromethane solution or dispersion of the polyradical was transferred onto a highly oriented pyrolytic graphite, and the solvent was carefully blotted off by air-drying. Tapping mode AFM using a Si probe (type TESP) was applied to estimation of the horizontal distance of particles. The size and the shape of probe are known to affect AFM images, therefore, the same probe was used for all measurements. The MFM images were presented by an amplitude or phase shift acquired with the Lift Mode technique. ${ }^{8}$ The scan lift height was controlled by the scanning parameters, assuming that the sensitivity of the cantilever was $0.03 \mathrm{~V} \mathrm{~nm}^{-1}$. A commercially available MFM probe (type MESP) coated with a ferromagnetic $\mathrm{CoCr}$ alloy possessing a magnetic moment of $1 \times 10^{-13} \mathrm{emu}$ was used, which was magnetized in the direction perpendicular to the sample surface.

The light scattering measurement was performed with a Coulter N4SD submicron particle analyzer. The scanning electron microscope used was a S2500CX (Hitachi) for observing the polyradical particle. The molecular weight of the polymers was estimated by GPC (polystyrene gel column, eluent THF, polystyrene calibration).

\section{RESULTS AND DISCUSSION}

It is well known that nm- or sub- $\mu$ m-sized and monodispersed polymer particles are obtained by a dispersion and emulsion polymerization. However, the obtained particles are covered with a stabilizer and an emulsifier that often results in a low yield in the following chemical modification of the particles. We used, in this study, an emulsifier-free emulsion terpolymerization of methacrylic acid, methyl methacrylate, and methylenebisacrylamide to obtain the particles. The feed composition of the methacrylic acid (1-30\%), methyl methacrylate as an oil-phase formation agent (94-65\%), and methylenebisacrylamide as a bifunctional crosslinker (5\%) through the emulsifierfree emulsion polymerization was first examined to obtain the poly(methacrylic acid) particle with a desirable size and applicable to the following reactions to introduce the TEMPO moiety. The more methacrylic acid was subjected to the polymerization, the more carboxyl groups were introduced into the particle, and a stable dispersion was maintained up to $30 \%$ of the methacrylic acid content. Next the carboxyl function of the particle was treated with an excess amount of thionyl chloride to yield the acid chloride, followed by 4-amino-2,2,6,6-tetramethylpiperidine-1oxyl in dichloromethane solution in the presence of tri- 
ethylamine. The introduction of TEMPO groups into the particle and its amount were confirmed by elemental analysis. The determined TEMPO amount was $c a$. 1.4 times larger than that from SQUID measurements shown below (e.g., $1.5 \mathrm{mmol} \mathrm{g}^{-1}$ from elemental analysis corresponded to $1.1 \mathrm{mmol} \mathrm{g}^{-1}$ from magnetic measurement).

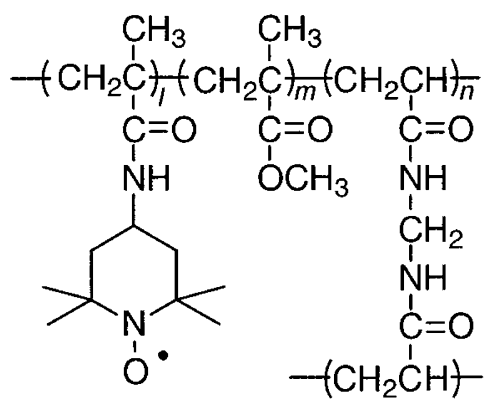

Chart 1.

Light scattering measurement on the particle dispersion in dichloromethane indicated, for example, an average diameter of $400 \mathrm{~nm}$ and a narrow distribution $( \pm 60 \mathrm{~nm})$ for the TEMPO sample derived from the particle prepared by the polymerization of methacrylic acid (20\%), methyl methacrylate (75\%), and methylenebisacrylamide (5\%). A dilute dichloromethane dispersion of the particle was transferred to a highly oriented pyrolytic graphite surface, and was subjected to AFM. An approximate $280 \mathrm{~nm}$ diameter was estimated for the polyradical particle. AFM gave a slightly smaller diameter than that by the light scattering, probably because of the lack of swelling or a solvent-free sample state for the AFM measurement.

The spin concentration and persistency of the polyradical particle were estimated by SQUID measurements. The magnetic susceptibility data for all samples obeyed the Curie-Weiss law with a small Weiss constant of less than $-1 \mathrm{~K}$. For four kinds of polyradical particles with almost the same diameter of ca. $300 \mathrm{~nm}$, the spin concentrations $\left(\mathrm{mmol} \mathrm{g}^{-1}\right)$ of 1.1 , $0.85,0.60$, and 0.17 were estimated from the slope of the plots in each case. These values correspond to the maximum yield of $c a .50 \%$ for the methacrylic acid residues in the particle. All polyradical particles prepared were fully stable at room temperature under atmospheric conditions for at least 6 months.

A direct one-step preparation of the desired polyradical particle was tried by the anionic dispersion polymerization of the nitroxide monomer, 4-methacryloyloxy2,2,6,6-tetramethylpiperidine-1-oxyl (MOTMP). It has been reported that both the radical and cationic polymerizations of a nitroxide-bearing vinyl monomer did not proceed but the anionic polymerization of the ni- troxide monomer, e.g., MOTMP, directly gave a nitroxide polyradical. ${ }^{9}$ MOTMP was anionically polymerized in hexane with sec-butyllithium as an initiator in the presence of polystyrene-block-polybutadiene as the stabilizer. Immediately after the addition of the initiator, there appeared a light orange turbidity, which indicated the progress of the polymerization and the particle formation. GPC measurement of the resulting polymer dissolved in THF indicated the polyradical formation with a molecular weight of $M_{\mathrm{n}}=3.2 \times 10^{3}$. Its spin concentration estimated from the SQUID measurement was $2.0 \mathrm{mmol} \mathrm{g}^{-1}$. This spin concentration was twice that of the highest one for the polyradical particle prepared by the polymer reaction. A scanning electron micrograph of the product revealed the formation of a relatively monodispersed particle with $c a .100 \mathrm{~nm}$ diameter and their aggregation. Thus it was concluded that the anionic dispersion polymerization of MOTMP was useful for preparing the corresponding polyradical particle with a high spin concentration in one step. However, the AFM images gave only the aggregate of particles and it was difficult to obtain an image of the dispersed single particle even by replacing the solvent species of the particle suspension and a stabilizer (e.g., polystyrene-block-poly(methyl methacrylate)) in the anionic dispersion polymerization. Therefore, this polyradical particle could not be subjected to the subsequent MFM measurement.

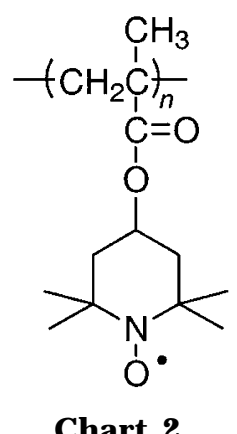

Magnetic force microscopy (MFM) is a scanning probe technique and a powerful tool for sub- $\mu \mathrm{m}$ magnetic imaging. ${ }^{10}$ The technique is, from an instrumental point of view, very closely related to AFM except for the use of a magnetized probe. The microscope's cantilever equipped with the ferromagnetic probe achieves a local magnetostatic interaction between the probe and a magnetic sample on the substrate or the sample surface. The first scan is in a tapping mode to recognize the surface or the shape of the sample, that is, AFM with the ferromagnetic probe. After lifting the probe to a certain height, the second scan in the same area uses the non-contact mode, and the magnetic gradient of the sample is detected by measuring the deflection of the 
cantilever equipped with the ferromagnetic probe in the static mode of MFM operation. In this study, our sample is paramagnetic and has a weak magnetic moment relative to ferromagnetic materials at which the MFM has generally aimed and been utilized, therefore, we set the sample holder on a powerful magnet to induce the magnetic vector of the sample. The magnet consists of a NdFeB alloy, with which a multimode SPM scanner AS-130 (J) for the Nanoscope IIIa is equipped. The effective external magnetic field at the sample position through a stainless holder was estimated to be ca. $80 \mathrm{G}$ using a Gaussmeter, which seemed to be sufficient to affect the paramagnetic sample in this study. The polyradical sample would sense an adequate external magnetic field, and the ferromagnetic MFM probe would respond to a change in the magnetic permeability at the position of the paramagnetic polyradical sample.

Dilute dichloromethane solution of the linear polyradical, poly(4-methacryloylamino- or 4-methacryloyloxy-2,2,6,6-tetramethylpiperidine-1-oxyl) as a control, was transferred to a graphite surface and subjected to AFM followed by MFM. Both images were obtained at ambient conditions or under air at room temperature. The MFM image appeared exactly on the molecular positions detected by the AFM image. However, the disadvantage of the linear polyradical was unsettled images, which strongly depended on the surface deposition process or evaporation process of the solvent, solvent species, and so on. These often include ellipse images with a broad distribution of diameter consisting of the aggregation of many molecules, taking into account the molecular weight of the polymer.

This result was in contrast to the polyradical particle with a controlled and monodispersed diameter. A clear particle or dot shape is observed for the polyradical particle, which was not affected by the deposition process on the graphite substrate. The AFM gave $10^{2} \mathrm{~nm}$-sized dot images of the single polyradical particle (Figure 1). The following MFM clearly indicated magnetic gradient responses exactly on the same single polyradical particle positions. The three-dimensional representation of the MFM topography demonstrates "holes" ascribed to the polyradical particle. By switching the drive frequency of the cantilever from higher to lower against the resonance frequency, the MFM image was inverted, which supported the magnetic response of the observed images and denied a noise image caused by surface information in the AFM.

Figure 1 shows the effect of the radical or spin concentration of the particle on the MFM image. For all measurements, the same apparatus and the same cantilever were used, and the scan lift height or the distance between the probe and the sample surface was

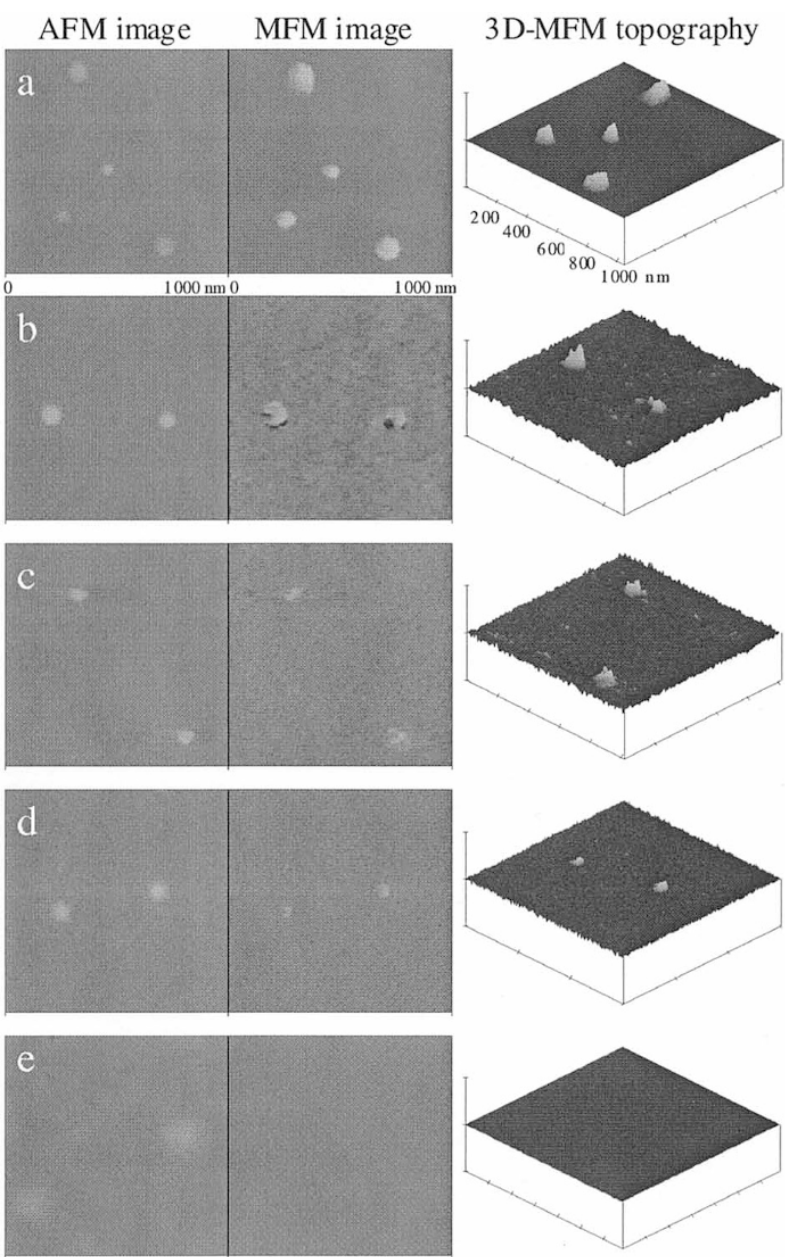

Figure 1. Effect of the spin or radical concentration of the polyradical particles. (left) AFM, (middle) MFM images obtained by detecting phase shifts in the cantilever oscillation caused by attractive forces acting on the ferromagnetically coated probe, and (right) three-dimensional representations of the MFM images of the polyradical particles with different spin concentrations $\left(\mathrm{mmol} \mathrm{g}^{-1}\right)$ (a) 1.1, (b) 0.85 , (c) 0.60 , (d) 0.17 , and (e) 0 .

maintained at $30 \mathrm{~nm}$. The intensity of the MFM image, that is, the magnitude of magnetic gradient decreased with a decrease in spin concentration of the polyradical particle. The magnetic gradient response was not observed for the particles without spin, which indicated that the contribution of diamagnetism to the probe at a certain height was almost the same in this series of study. The MFM vertical scale could be, in principle, represented by a force which is related to the detailed magnetic properties of the probe such as the absolute value of the effective magnetic moment and its hysteresis loop, the spring constant of the cantilever upon measurement, and the scan lift height, but it was given as an arbitrary unit here.

The MFM of the particle with a low spin concentration of $0.17 \mathrm{mmol} \mathrm{g}^{-1}$ gave an interesting hollow dot image when the scan lift height was adjusted to $35 \mathrm{~nm}$ (Figure 2). On this particle sample, the radical group 

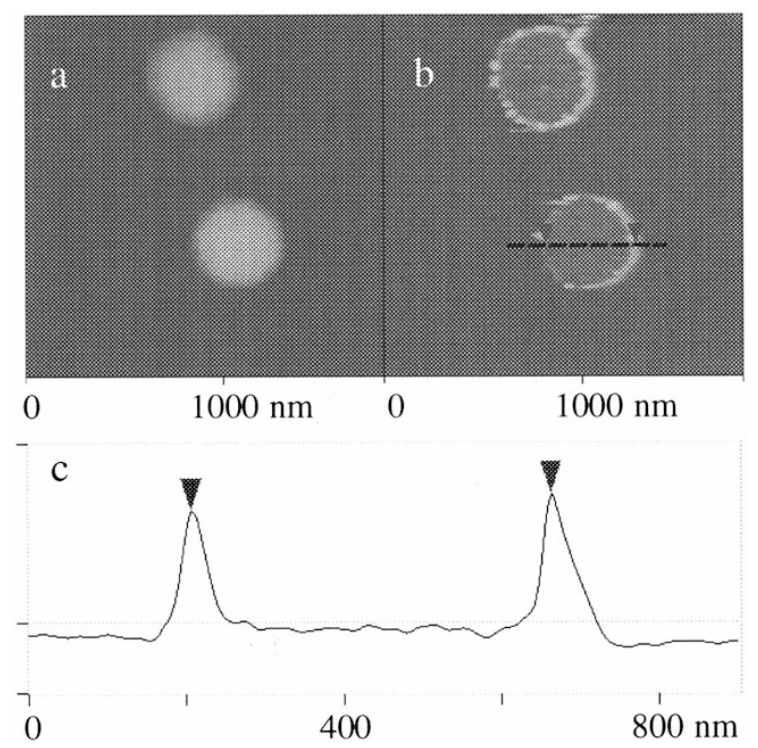

Figure 2. MFM hollow dot image of the polyradical particle with low spin concentration. (a) AFM, (b) MFM, and (c) cross sectional view of the MFM image.

was introduced by the polymer reaction and the spins are homogeneously distributed only over the particle surface where the reaction initially occurred but not inside. The magnetic response as the deflection of the cantilever is based on the second differential of the magnetic field in the vertical direction. A strong magnetic gradient is detected on the perimeter of the particle but is very weak in the interior. This consideration could explain such a hollow dot image.

It is concluded that MFM is an effective tool to detect the very weak magnetic signal of radical polymers and that a nm-sized polyradical particle is a new material of molecular-based magnetic dots potentially applicable to multiple representations of magnetic information.
Acknowledgment. This work was partially supported by a Grant-in-Aid for Scientific Research (No. 13031072) and COE Research "Molecular NanoEngineering" from MEXT, Japan. T. M. acknowledges the Research Fellowships of the Japan Society for the Promotion of Science for Young Scientists.

\section{REFERENCES}

1. a) F. J. Himpsel, J. E. Ortega, G. J. Mankey, and R. F. Willis, Adv. Phys., 47, 511 (1998).

b) J.-Q. Wang, L. M. Malkinski, Y. Hao, C. A. Ross, J. A. Wiemann, and J. O'Connor, Mater. Sci. Eng., B, 76, 1 (2000). c) J. Lohau, S. Kirsch, A. Carl, and E. F. Wassermann, Appl. Phys. Lett., 76, 3094 (2000).

2. D. A. Tomalia, H. Baker, J. Dewald, M. Hall, G. Kallos, S. Martin, J. Roeck, and P. Smith, Polym. J., 17, 117 (1985).

3. H. Kawaguchi, Prog. Polym. Sci., 25, 1171 (2000).

4. a) P. M. Lahti, "Magnetic Properties of Organic Materials", Marcel Dekker, Inc., New York, N.Y., 1999.

b) K. Ito and M. Kinoshita, "Molecular Magnetism - New Magnetic Materials", Kodansha and Gordon and Breach Science Publishers, Tokyo and Amsterdam, 2000.

5. H. Nishide, T. Ozawa, M. Miyasaka, and E. Tsuchida, J. Am. Chem. Soc., 123, 5942 (2001).

6. a) T. Kurosaki, K. W. Lee, and M. Okawara, J. Polym. Sci., Polym. Chem. Ed., 10, 3295 (1972).

b) M. Kamachi, M. Tamaki, Y. Morishima, S. Nozakura, W. Mori, and M. Kishita, Polym. J., 14, 363 (1982).

7. J.-S. Wang and K. Matyjaszewski, J. Am. Chem. Soc., 117, 5614 (1995).

8. V. Elings and J. Gurley, U.S. Patent 5308 974, Digital Instruments, Santa Barbara, CA, May 3, 1994.

9. a) O. H. Griffith, J. F. Keana, S. Rottschaefer, and T. A. Warlick, J. Am. Chem. Soc., 89, 5072 (1967).

b) J. Allgaier and H. Finkelmann, Makromol. Chem., Rapid Commun., 14, 267 (1993).

10. a) L. A. Bottomley, Anal. Chem., 70, 425R (1998).

b) U. Hartmann, Annu. Rev. Mater. Sci., 29, 53 (1999). 